\title{
Exercise training response heterogeneity: statistical insights
}

\author{
Greg Atkinson $^{1} \cdot$ Philip Williamson ${ }^{1}$ - Alan M. Batterham ${ }^{1}$
}

Received: 23 October 2017 / Accepted: 24 October 2017 / Published online: 15 November 2017

(C) Springer-Verlag GmbH Germany, part of Springer Nature 2017

Keywords Exercise training · Individual differences · Precision medicine $\cdot$ Trial-to-trial variability

\section{Abbreviation \\ MCID Minimal clinically important difference}

To the Editor: We would like to thank Dr Sparks for her comprehensive review of research on individual differences in the responses to exercise training [1]. It was especially helpful to see some of the statistical issues touched upon in this review [2]. We believe that these statistical factors are crucial for answering the fundamental question of whether there are true and clinically important individual differences in the response to exercise. By 'true', we mean individual response differences that are not merely random trial-to-trial variability in disguise. By 'clinically important', we mean individual differences that exceed a well-rationalised minimal clinically important difference (MCID). We also maintain that, in the 'roadmap' for researching this topic, true and clinically relevant individual response differences should be confirmed empirically before any moderators and mediators of the exercise response are explored [3].

The definition of 'non-response' was given in the review by Lauren Sparks [1] as, 'the lack of a difference between a control and a treatment condition with respect to a specific

Greg Atkinson

greg.atkinson@tees.ac.uk

1 Health and Social Care Institute, School of Health and Social Care, Constantine Building, Southfield Road, Teesside University, Middlesbrough, Tees Valley TS1 3BA, UK variable'. This definition implies that researchers can identify non-responders simply by looking at their data from a twocondition (control and exercise) experiment and concluding that those participants with a treatment - control difference close to zero or in the opposite direction to that expected are identified as 'non-responders' (the latter are sometimes labelled 'adverse responders'). The fallacy of this approach was hinted at by Barker and Schofield [2], but the full implications of this issue were not explicitly described. We have provided a full account of the pitfalls in non-responder identification $[3,4]$, and think that they can complement the useful review by Sparks [1].

An observed response is comprised of the true response as well as random trial-to-trial within-individual variability [3]. Therefore, observed non-response to exercise, or any other treatment, does not necessarily mean that there has been a true non-response. In Fig. 1, we present some simulated data that appears to show that individual participants differed substantially in terms of their acylated ghrelin response to exercise. It is well documented that exercise causes a reduction in the mean concentration of acylated ghrelin [5]. For the simulated data in Fig. 1, the mean \pm SD reduction in acylated ghrelin was $18.1 \pm 23.1 \mathrm{pg} / \mathrm{ml}(95 \%$ CI 1.6, 34.7). Nevertheless, it appears as though there are four non-responders in this sample of ten participants, according to the definition provided in $\mathrm{Dr}$ Sparks' review [1].

In reality, true individual differences in response to exercise do not exist in the data presented in Fig. 1. In our simulation we subtracted exactly $25 \mathrm{pg} / \mathrm{ml}$ of ghrelin from each participant's control condition measurement. We then added the component of a typical magnitude of random trial-to-trial variability. The trial-to-trial correlation coefficient was 0.77 (95\% CI $0.27,0.94)$. This random variability in biological measurements from day-to-day or week-to-week is always present and uncontrollable. Importantly, it is this component of 


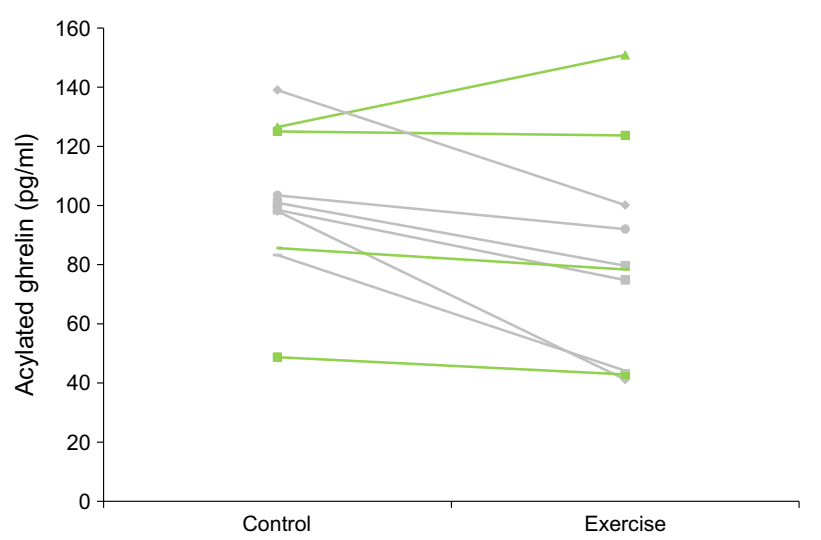

Fig. 1 Apparent individual differences in the acute response of acylated ghrelin to a bout of exercise. Apparent non-responders are indicated by the green lines. In reality, there are no true individual differences in response in these simulated data. The apparent individual differences are due solely to the influence of trial-to-trial variability in ghrelin concentrations

variance on its own that makes it look as though individual differences in exercise response exist, when this may not necessarily be so. Naturally, this component of variance can also influence the observed mean difference relative to the true mean difference, as it does in our data used to generate Fig. $1(18.1 \mathrm{pg} / \mathrm{ml}$ vs $25 \mathrm{pg} / \mathrm{ml}$, respectively).

For repeated trial studies, the optimal design for quantifying individual response differences is the replicate crossover design [6]. Here, the control and exercise conditions are actually administered to each participant at least twice, with the sequence of the four trials randomised. This design allows the researcher to derive the intervention $\times$ participant interaction term from the statistical model [6], thereby enabling them to isolate the true individual differences in response to exercise. To our knowledge, this design has not yet been used in an exercise context, although we have several such studies ongoing at present.

Within-individual variability in the measured outcome also causes problems when interpreting the results of longer-term exercise training studies $[3,4]$. In many of these studies, a control group is either not present or discarded in the data analysis. Plots of individual differences in baseline to follow up data are commonly presented for the exercise training arm only, as demonstrated in the upper graph of the figure in the review by Sparks [1]. Nevertheless, a very similar graph can usually also be plotted for the changes in baseline to follow up for the control group. These data are seldom presented but are crucial for ascertaining whether there are clinically relevant true individual differences in training response [3].

In a parallel group study, true individual differences in exercise response are present only if the SD of change is substantially larger in the exercise group than the control group. If not, the apparent individual differences in response are nothing but baseline-to-follow-up within-individual variability, just as in Fig. 1. This random variability can be large if there are many weeks $(>6)$ between baseline and follow up in the study, and such longer-term durations of follow-up are commonly selected in studies. We also recently presented a critical review of a selected sample of exercise training studies; studies were included if the relevant data were reported and if the outcome was change in $\dot{V} \mathrm{O}_{2 \text { peak }}$ [4]. We found that very few studies included data from a control group in their analyses. For those studies that had a control group, we found little evidence that the difference in the SD of changes between intervention and control was clinically important, relative to an MCID of 1 metabolic equivalent (MET).

We maintain that ascertaining whether there are true individual differences in the responses to exercise that are large enough to be clinically relevant is a crucial platform for precision medicine. If these individual differences in response are found to be not clinically important, we question the need to proceed to explore individual moderators and mediators of response. Such explorations could be wasteful in terms of participant time as well as money from a funding body. We have also highlighted in this letter that an understanding of the impact of trial-to-trial or baseline-to-follow up variance is crucial for making robust inferences about individual response differences.

Duality of interest The authors declare that there is no duality of interest associated with this manuscript.

Contribution statement All authors were responsible for drafting the article and revising it critically for important intellectual content. All authors approved the version to be published.

\section{References}

1. Sparks LM (2017) Exercise training response heterogeneity: physiological and molecular insights. Diabetologia 60:2329-2336

2. Barker RJ, Schofield MR (2008) Classifying individuals as physiological responders using hierarchical modeling. J Appl Physiol 105: $555-560$

3. Atkinson G, Batterham AM (2015) True and false inter-individual differences in the physiological response to an intervention. Exp Physiol 100:577-588

4. Williamson PJ, Atkinson G, Batterham AM (2017) Inter-individual responses of maximal oxygen uptake to exercise training: a critical review. Sports Med 47:1501-1513

5. Schubert MM, Sabapathy S, Leveritt M, Desbrow B (2014) Acute exercise and hormones related to appetite regulation: a meta-analysis. Sports Med 44:387-403

6. Senn S, Rolfe K, Julious SA (2011) Investigating variability in patient response to treatment - a case study from a replicate cross-over study. Stat Methods Med Res 20:657-666 